\title{
LA TAREA DE AUTO-CONSUELO COMPASIVO EN TERAPIA FOCALIZADA EN LA EMOCIÓN
}

\section{THE COMPASSIONATE SELF-SOOTHING TASK IN EMOTION-FOCUSED THERAPY}

\author{
Ciro CARO ${ }^{1}$ Y TERESA HORNILLOS $^{1}$
}

Cómo referenciar este artículo/How to reference this article:

Caro, C. y Hornillos, T. (2015). La tarea de auto-consuelo compasivo en Terapia Focalizada en la Emoción [The Compassionate Self-Soothing Task in Emotion-Focused Therapy]. Acción Psicológica, 12(2), 73-94. doi: http://dx.doi.org/10.5944/ap.12.2.15808

\section{Resumen}

El objetivo de este artículo es poner a disposición de los terapeutas y de los equipos de investigación clínica en psicoterapia, el conocimiento actualizado sobre la Tarea de Auto-consuelo Compasivo de la Terapia Focalizada en la Emoción (TFE). Para los terapeutas humanistas o integradores que guían su intervención mediante marcadores, y que trabajan en el nivel experiencial, este texto pretende ser una guía formativa inspiradora a la vez que detallada. Paralelamente, para los investigadores comprometidos con el desarrollo de
\end{abstract}

la TFE, el texto es propuesto como un modelo, que puede ser utilizado para homogeneizar e incrementar la validez de las intervenciones de facilitación del autoconsuelo, en estudios clínicos en los que este proceso sea una variable relevante. Primero se encuadra la Tarea de Auto-consuelo Compasivo en el marco conceptual de la TFE, se fundamenta racionalmente el trabajo terapéutico con la auto-compasión, y se informa acerca de la evidencia empírica disponible en este campo. Después se explica la tarea en términos del modelo de proceso resultado de la investigación y de la observación clínica: (1) descripción del marcador, (2) especificación de las operaciones del cliente y del terapeuta y (3) propuesta de un estado final relacionado

Correspondencia: Ciro Caro. Depatamento de Psicología, Universidad Pontificia Comillas de Madrid, España.

Email: cirocaro@comillas.edu

${ }^{1}$ Universidad Pontificia Comillas de Madrid, España.

Recibido: 13 de septiembre de 2015

Aceptado: 30 de octubre de 2015 
con un micro-resultado terapéutico típico dentro de la sesión. Además se presenta la correspondiente escala de resolución del proceso que conduce a ese estado final. Y por último, se hace una serie de sugerencias acerca del modo óptimo de utilizar este material y se ofrecen algunos recursos didácticos y de entrenamiento complementarios.

Palabras clave: auto-consuelo; compasión; Terapia Focalizada en la Emoción; proceso terapéutico; formación en Psicoterapia.

\begin{abstract}
The aim of this paper is to make available to the therapists and to the psychotherapy clinical research teams an update, on the Compassionate Self-soothing Task in Emotion-Focused Therapy (EFT). For the humnanistic or integrative therapists that use marker-guided interventions and for those who work at an experiential level, this writing is aimed to be an inspiring and detailed training guide. On the other hand, for those researches committed to the EFT development, the text is proposed as a model that may be used to homogenize and to increase the validity of the selfsoothing facilitation interventions, in those studies in which such process is a relevant variable. In the first place, we present the Compassionate SelfSoothing Task within the EFT's conceptual framework; then we give a rationale for the therapeutic work with self-compassion, and finally we present the available empirical evidence in the field. The task is also explained in terms of the process model resulting from clinical research and observation: (1) marker description, (2) client's and therapist's operations specification, (3) proposal of an end state related to a typical in-session therapeutic micro-outcome. In addition the resolution scale of the process that leads to the end state is also presented. Finally, we make a series of suggestions on how to use this material, and provide some supplementary didactic and training resources.
\end{abstract}

Keywords: self-soothing; compassion; Emotion-Focused therapy; therapeutic process; Psychotherapy training.

\section{Encuadre en la Terapia Focalizada en la Emoción}

La Terapia Focalizada el Emoción (TFE) es un enfoque procesual-experiencial de psicoterapia, que incorpora los fundamentos teóricos así como las prácticas de la Terapia Gestalt, la Terapia Centrada en la Persona y la Terapia Experiencial junto con otras aproximaciones humanistas y existenciales (Elliott, Watson, Goldman y Greenberg, 2004; Greenberg, Rice y Elliott, 1993). Asume la visión y valores de la terapias humanistas y revisa sus fundamentos epistemológicos a partir del constructivismo, la neurociencia y teoría moderna de la emoción (Greenberg y Pascual-Leone, 1995; Greenberg, Rice y Elliott, 1993; Lane, Ryan, Nadel y Greenberg 2015). Así mismo, aporta una metodología de investigación que no violenta la naturaleza experiencial y dialógica de la terapia, y que permite generar evidencia empírica a través de la investigación de procesos de cambio y su relación con los resultados terapéuticos (Angus, Watson, Elliott, Schneider y Timulak, 2015; Elliott, 2010a; Greenberg, 1994; Pascual-Leone y Greenberg, 2009).

La TFE se centra en ayudar a los clientes a explorar y modificar sus experiencias emocionales y sus estructuras subyacentes (esquemas de emoción), lo cual implica el trabajo con una serie de constelaciones de percepción, emoción, cognición, experiencia corporal, conducta y significado, que dan forma a las reacciones de la persona (Elliott et al., 2004), y constituyen su ser-ahí como procesos de auto-organización y desorganización del sí mismo (Caro, 2005; Whelton y Greenberg, 2000). Así, los terapeutas distinguen entre emociones primarias adaptativas -como la tristeza ante la pérdida o el miedo ante la amenaza-, que son reacciones inmediatas a situaciones de la vida de los clientes por una parte, y emociones primarias desadaptativas por otra, que también son 
reacciones inmediatas pero basadas en esquemas de emoción disfuncionales formados en el pasado en situaciones traumáticas, y que ya no resultan útiles. Igualmente distinguen las emociones secundarias -como la rabia, la vergüenza o la ansiedad-, que son respuestas que se producen ante emociones primarias. Por ejemplo, el trabajo terapéutico con la ansiedad social implica ayudar a los clientes a reconocer sus emociones secundarias familiares (como la ansiedad) y transformar sus emociones primarias desadaptativas (como la vergüenza crónica) emociones primarias (como la tristeza ante la pérdida de conexión o la rabia y el enfado ante un trato injusto recibido por otros). A través de este proceso se desarrollan esquemas de emoción alternativos, que permiten a la persona auto-organizarse con una sensación de mayor conexión con el sí mismo y con los otros (e.g., orgullo, curiosidad, o autocompasión).

La investigación clínica en el marco de la TFE ha permitido especificar diversos procesos terapéuticos en términos de tareas (con un marcador que indica la disponibilidad del cliente para implicarse diferencialmente en cada tarea, un mapa de proceso con las operaciones del terapeuta y los procesos del cliente, y un estado final). Estas tareas constituyen procesos de cambio específicos, que permiten modificar los esquemas de emoción disfuncionales. Algunas están basadas en la empatía, otras son de carácter relacional, otras son propiamente experienciales, varias se caracterizan por facilitar el reprocesamiento narrativo de una experiencia, y finalmente hay un grupo de ellas que son tareas de representación o dramatización, también denominadas enactivas (Elliott, et al., 2004), las cuales son un desarrollo de las técnicas de trabajo con las dos sillas de la Terapia Gestalt. El uso terapéutico de las sillas fue propuesto para ayudar a los clientes a acceder a emociones y aspectos del sí mismo previamente no reconocidos y así poder expresarlos (Elliott et al., 2004; Greenberg, 2002). Por otra parte, aunque estas representaciones son frecuentemente facilitadas utilizando sillas, también otros objetos como marionetas o las propias manos, así como la imaginación (imaginarte a ti mismo como un niño de 5 años) pueden ser empleados. Las representaciones en TFE están basadas en dos tipos principales de trabajo con las sillas (Elliott et al., 2004; Greenberg y Watson, 2005): el diálogo con las dos sillas para escisiones por conflicto o po- laridades dentro del sí mismo del cliente (referido en la terapia Gestalt como "diálogo interno"), y el trabajo con la silla vacía para asuntos inacabados o sentimientos negativos duraderos hacia un otro significativo (referido en Terapia Gestalt como "diálogo interno"). Sin embargo, más recientemente, otra tarea de representación que implica la técnica del diálogo externo con las dos sillas ha sido introducida en el marco de investigación y práctica de la TFE, bajo la denominación de "Tarea de Autoconsuelo Compasivo" (TAC) (Elliott, 2012; Goldman y Fox, 2010; Goldman y Greenberg, 2010, 2013; Sutherland, Peräkylä, y Elliott, 2014), y de ella se ocupa este trabajo.

En su revisión de los modos en que el terapeuta focalizado en la emoción puede ayudar a los clientes a acceder a emociones adaptativas alternativas, así como a necesidades primarias en el proceso de coaching emocional, Greenberg (2002) puso de relieve que en ocasiones, las personas necesitan no sólo transformar sus emociones sino también aprender a regularlas. Entonces revisa tres grades estrategias de regulación emocional, junto a las que presenta su experiencia con ejercicios de auto-consuelo en la sesión: (a) las habilidades de conciencia plena -mindfulness- (Kabat-Zinn, 1993, 2005) que pueden ser enseñadas a los clientes; (b) los aportes de Marsha Linehan (1993) en su modelo de Terapia Dialéctico-Conductual, desarrollados para ayudar a los clientes con diagnóstico de Trastorno Límite de la Personalidad a través de una serie de técnicas de regulación emocional orientadas conductualmente, en las que también éstos pueden ser entrenados (De la Vega y Sánchez, 2013); y (c) la posibilidad de crear una distancia de trabajo respecto a la emoción, y de construir un espacio seguro al que retirarse en caso de necesidad. Frente a estas posibilidades, describe su experiencia con el autoconsuelo como método apoyado en un diálogo interno, adecuado para aquellas ocasiones en las que la persona expresa una intensa auto-condena y un fuerte autodesprecio, y parece incapaz de acceder a ninguna de sus capacidades para calmarse a sí misma. Además refirió que esta intervención puede ser especialmente útil en clientes que se autolesionan o que utilizan la automedicación para regular su emoción. 
El diálogo reproducido en la Tabla 1 ha sido reconstruido a partir del ejemplo propuesto por Greenberg (2002, p. 208), e ilustra brevemente esta manera de trabajar con el auto-consuelo en la sesión, que es tanto un precedente de la TAC en TFE, como una de las formas simples en las que, en ocasiones, este tipo de procesos son facilitados vivencialmente en la sesión.

\section{Fundamento racional del trabajo con el auto-consuelo y la auto-compasión en psicoterapia}

Los procesos de auto-consuelo son una potente fuente de experiencias emocionales correctoras, que cuando son integrados en la sesión como tareas terapéuticas, se unen al grupo de experiencias correctoras que Greenberg y Elliott (2012) consideran de carácter intrapersonal: aquellas en que la acción primordial implica a diferentes partes del sí mismo (p. 94). Por otra parte, el auto-consuelo puede ser vinculado al cada vez más popular concepto de auto-compasión (Bernard y Curry, 2011). Neff lo desarrolló a partir de la tradición filosófica budista y

Tabla 1

Facilitación básica del auto-consuelo reconstruido a partir de Greenberg (2002, p. 208)

C: $\quad$ Me gustaría que esa parte tan llorona de mí, simplemente se recuperara [volviendo los labios hacia dentro]. Mi madre me ignoraba y mi padre me manipulaba emocionalmente... iy qué! Lo único que tenía que haber hecho era dejar de lloriquear.

T: $\quad$ Imagina a una niña de ocho años aquí sentada. Su madre apenas la mira y pasa de hablarle. Su padre se apoya en ella cuando se siente mal, para compensar así todo el amor que no puede obtener de su mujer, pero la rechaza cuando no la necesita... ¿Cómo te imaginas que es todo eso para ella? [deja pasar unos segundos]. Date cuenta, y mira a ver ¿qué le dirías?

C: $\quad$ Sé que se sentirá muy sola, sin nadie. Y creo que se merece algo más.

T: $\quad$ Prueba a decírselo. ¿Puedes darle ahora algo de lo que ella necesitaba?

C: $\quad$ Sé que te sientes sola, y que te mereces algo más.

T: $\quad$ ¿Podrías hacer lo mismo con la niña que se siente sola dentro de ti? Reconocerle cómo se siente y el amor que necesita... ¿y luego tratar de darle algo de ese amor? Dile... porque tú sabes mucho de lo que ella vivía con tus padres...

C: $\quad$ ¡Ay...! [suspira]. A mamá parecías no importarle, y papá sólo te usaba según cómo se sintiera él, y dentro de ti sólo sentías que no le importabas a nadie... y te sientes sola aunque haya gente a tu alrededor... porque dentro te falta todo ese amor que ellos no te daban.

T: $\quad$ Dáselo... por ejemplo dile "Yo quiero darte algo de ese amor".

C: $\quad$ Pero yo tengo ese amor y... te quiero... porque te veo tan pequeña y tan indefensa, tratando de comprender por qué mamá no te hace caso [con lágrimas]. 
El auto-consuelo y las formas de auto-compasión (moldeadas o instruidas) son prácticas psicoterapéuticas que encuentran un creciente reconocimiento en el marco conceptual de las terapias de conducta contextuales o de tercera generación (Pérez Álvarez, 2014). En ellas, podrían inscribirse como procedimientos que inicialmente responden al principio de aceptación (abandonar la lucha contra los síntomas) y que después se desplegarían como procesos guiados por el principio de activación (reorientación de la vida en la dirección de los valores y de las cosas que para la persona son importantes, entre las cuales puede hallarse el sí mismo). Así, Gilbert $(2009,2015)$ desarrolló su modelo de Terapia Centrada en la Compasión (TCC) tras observar que las personas con historias cronificadas de vergüenza, autocrítica y odio hacia sí mismas, con frecuencia, al ser tratadas con Terapia Cognitivo-Conductual afirmaban entender la lógica de los pensamientos alternativos, pero seguían sintiéndose mal. Para explicar esto, postuló que tales pacientes sufren una dificultad para acceder a un sistema emocional, que en los mamíferos evolucionó junto con los sistemas de cuidado, y que sería responsable de los sentimientos de tranquilidad, seguridad y conexión, lo cual mantendría el problema. Por ello planteó la TCC en términos de una lógica estratégica, por la cual habría que ayudar a las personas a distanciarse de sus pensamientos, sentimientos y problemas, para después tratarse a sí mismas con más compasión y amabilidad, desarrollando en este segundo tiempo el fenotipo de la conducta afiliativa. En su marco evolucionista Gilbert (2015) propone una teoría de las "mentalidades sociales", que serían formas de organización mental de base biológica, que capacitan a los individuos para procurarse y formar determinados tipos de relación, y estarían asociadas a determinadas metas y motivos bio-sociales. Tales organizaciones mentales funcionarían modularmente (e.g., simpatía, cuidado o agresión), y se sucederían activándose unas y desactivando otras. Por ello la terapia debería servir para desactivar algunos tipos de mentalidad en favor otras, entre las que destaca la "mente compasiva", que sería lo contrario de la "mente amenazante". Para eso propone que el paciente comprenda el origen y la función de la autocrítica (incluidas sus asociaciones con orígenes tempranos), y sugiere imaginarla de forma externalizada o incluso recurrir a un trabajo de diálogo con las sillas.
Después plantea cómo el terapeuta puede ayudar al cliente a desarrollar una mente compasiva, que funcione como una alternativa para cumplir las mismas funciones, pero basada en un sistema emocional diferente. Tal desarrollo es planteado por Gilbert (2015) como un "entrenamiento compasivo de la mente" (p. 154), y constituye la fase activa de la intervención.

Por otra parte, las terapias humanistas y experienciales se ocuparon de este asunto (la auto-relación compasiva) desde el principio, particularmente en el marco de la investigación de procesos de cambio en psicoterapia, en la que se apoyó el desarrollo de sus modelos, fuertemente enraizados en la dialéctica de contrastación de sus hipótesis y de refinamiento de la teoría. Así -y de acuerdo con el recuento somero de Neff (2003a) - contamos con varios antecedentes de estos planteamientos: (a) ya Maslow en la década de los 60 resaltó la importancia de ayudar a las personas a aceptar y a generar compasión hacia su propio dolor y sus propios defectos, como algo necesario para su crecimiento y para promover lo que denominó "Percepción B hacia uno mismo", caracterizada por ser no evaluativa, perdonadora, amorosa y aceptadora del ser (Being); (b) Carl Rogers propuso la actitud de "mirada positiva incondicional" como uno de los tres pilares de la Terapia Centrada en la Persona (TCP), la cual debía ser desarrollada por el paciente a través de la interacción con el terapeuta, y que para algunos autores posteriores es equivalente a una forma de amor (Brazier, 1997), y (c) Snyder sugirió que una de las metas de la terapia debería ser el desarrollo de un "empatizador interno". Además, Gendlin (1997, p. 282). desarrolló la noción "cliente del cliente" para referirse a una observación bien contrastada en la TCP y que consistía en una "distinción dentro de la persona", por la cual la experiencia se desdobla en dos aspectos: las nuevas vivencias emergentes, y las necesidades de los clientes respecto a estas nuevas experiencias (e.g., la aceptación y validación que puede ofrecer el terapeuta hacia la experiencia actual y el trabajo de aceptación de la propia experiencia que le corresponde al cliente).

La auto-compasión y el auto-consuelo también pueden ser considerados desde la perspectiva más general de la "regulación emocional", entendida ésta como el conjunto de procesos mediante los cuales una persona influye 
sobre diversos aspectos de sus emociones (atención, manejo de intensidad, duración de la activación, naturaleza y significado de los estados afectivos, modo de expresión, etc.), cuando se enfrenta con una situación estresante o angustiante (Gross, 1999; Thompson, 1994). A este respecto, Neff (2003a) propone que la autocompasión puede ser vista como una estrategia de afrontamiento emocional útil, así como un aspecto de inteligencia emocional, y en términos propiamente psicoterapéuticos, Greenberg (2002) planteó el autoconsuelo compasivo como una intervención específica orientada a revertir estados emocionales desregulados.

Sin embargo, la autocompasión también puede ser articulada en la sesión de terapia en el momento oportuno, como un proceso de auto-consuelo transformador de carácter dialógico vivido en primera persona, y como una forma de cambio emocional específica que no se limita a la reversión de un estado desregulado. Esto último es lo que se pretende con la TAC en TFE, de modo que la reestucturación del sí mismo en el nivel esquemático dé lugar a nuevas formas de auto-organización adaptativas (Caro, 2005; Goldman y Greenberg, 2014; Whelton y Greenberg, 2000; ver apartado sobre La dificultad de procesamiento subyacente más adelante). Por ello, y a diferencia de lo que sucede con las formas de autocompasión entrenadas como prácticas discretas -ya sea que fomenten una mentalidad o que desarrollen una estrategia de afrontamiento saludable-, en las que contar con diversos ejercicios y guías para su enseñanza puede ser suficiente, en el entrenamiento en modos de intervención experienciales como estos, son necesarios recursos formativos que ayuden a los terapeutas a trabajar momento a momento con la experiencia del cliente, en términos del tipo de coaching emocional al que nos referíamos anteriormente (Greenberg, 2002).

\section{Evidencia empírica disponible}

Aunque la auto-compasión parece ser central en el bienestar psicológico (Neff, 2003a; Yarnell y Neff, 2013), el conocimiento acerca de los procesos terapéuticos y de las intervenciones encaminadas a promoverlo (Bernard y Curry, 2011; Sahar et al., 2012) es realmente incipiente, si bien se halla en expansión. Los medios más comúnmente utilizados, junto con los resultados terapéuticos obtenidos en la investigación sobre cada uno de ellos fueron revisados por Sutherland et al. (2014) y los sintetizamos en la Tabla 2. Una consideración global de estos estudios sugiere que las prácticas auto-compasivas son eficaces para modular los estados emocionales disfuncionales de los pacientes ya sea en contextos experimentales (en los que la práctica era facilitada como una intervención aislada y se observaba su efecto sobre una variable relevante), o bien en contextos propiamente clínicos (en los que la práctica se integraba en un proceso psicoterapéutico).

Goldman y Fox (2010) reportaron un análisis de tareas (A. Pascual-Leone y Greenberg y J. PascualLeone, 2009) inicial acerca del trabajo de auto-consuelo en TFE, en el cual identificaron el marcador como una incapacidad para tolerar y regular la angustia frente a una a estado emocional doloroso desregulado, o ante una poderosa necesidad existencial no satisfecha (e.g., una necesidad de amor o validación). Este análisis de tareas, así como la reflexión sobre los casos de terapia en los que se ha utilizado la TAC, han conducido al modelo de proceso que se describe más adelante, en el que se explica cómo sucede el cambio a lo largo de la tarea.

Por otra parte, Sutherland et al. (2014) realizaron un análisis conversacional de la TAC a partir de la transcripción de segmentos de varios casos (uno de los cuales se propone como material formativo en este trabajo). El análisis de la interacción entre cliente y terapeuta aportó una descripción detallada de los procesos conversacionales implicados en el logro del auto-consuelo. E inspirándose en el concepto de "marco de participación" propuesto por Goffman (1981), citado por Sutherland et al. (2014, p. 740), los autores consiguieron mostrar cómo terapeutas y clientes colaboran para moverse desde un "marco ordinario" en la conversación hasta un "marco de auto-consuelo" como acción o diálogo, y de vuelta al primero, usando 
Tabla 2

Estudios sobre métodos terapéuticos que utilizan la auto-compasión

Síntesis de estudios sobre métodos terapéuticos que integran herramientas y procesos de auto-compasión (extraído y elaborado a partir de Sutherland, Peräkylä y Elliott, 2014)

\section{Entrenamiento en mente compasiva \\ (compassionate mind training, CMT) \\ Uso de imaginería auto- compasiva}

\section{Estrategias de reducción del estrés basada en mindfulness y en meditación}

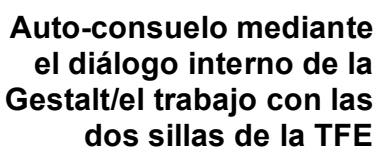

Gilbert, 2009; Gilbert y Irons, 2004; Gilbert y Procter, 2006

Desarrollada para fortalecer la auto-compasión entre individuos autocríticos. Se ha hallado que reduce significativamente la depresión, la vergüenza, la autocrítica, la ansiedad y otros síntomas después del entrenamiento.

\section{Gilbert y Irons, 2005; Gilbert y Procter, 2006}

Es un aspecto de la CMT que implica que los clientes visualicen a un otro "nutritivo cierra comillas y se les instruya para reclamar su presencia en las ocasiones de malestar o incluso para escribir cartas al sí mismo desde el punto de vista de esa figura.

Kelly, Zuroff y Shapira, 2009

Utilizaron un programa de ejercicios de imaginería y de auto-charla diarios durante dos semanas, y consiguieron reducir la vergüenza y las quejas sobre la piel en un grupo de pacientes afectados por acné.

\section{Lutz, Brefczynski-Lewis, Johnstone y Davidson, 2008}

Utilizando técnicas de neuro-imagen, hallaron que la meditación compasiva era eficaz para fortalecer el bienestar físico y psicológico de los individuos a largo plazo.

\section{Goldman y Fox, 2010}

Realizaron un análisis de tareas inicial sobre los procesos de auto-consuelo en la sesión en TFE.

\section{Shahar et al., 2012}

Mostraron que el trabajo con las dos sillas para la autocrítica condujo a un incremento significativo de la auto-compasión y a una reducción de la auto-crítica, la depresión y la ansiedad.

MacLeod, Elliott y Rodgers, 2012

Contribuyeron al apoyo de la eficacia de la TFE para la ansiedad social a través de un estudio de caso único, que incluía la Tarea de Auto-consuelo compasivo.

\section{Sutherland, Peräkylä y Elliott, 2014}

Realizaron un análisis conversacional de la Tarea de Auto-consuelo con las dos sillas en TFE, a partir de la transcripción de segmentos de sesiones de terapia procedentes de siete casos. varias prácticas de interacción: las instrucciones del terapeuta a los clientes, los modos específicos de secuenciar las acciones en la interacción, las explicaciones y justificaciones acerca de la importancia de la tarea de auto-consuelo, el uso de diferentes pronombres para distinguir entre discursos (e.g., el cliente vs. los agentes consoladores), o las correcciones del discurso del cliente y su reacción a diferentes respuestas de seguimiento (ajá, $m m m$, sí, bien...).
Además, también documentaron las prácticas conversacionales usadas por los clientes para lograr el auto-consuelo en términos de auto-elogio, revelación de cariño y preocupación, y ofrecimiento de consejo útil. Este estudio aporta una evidencia de tipo cualitativo, que permite establecer lazos entre los aspectos procesuales (que comprenden lo que hacen o dicen los terapeutas y los pacientes), y el tipo de micro-resultados dentro de la sesión, que se esperan del trabajo con la TAC. 


\section{El trabajo clínico con la Tarea de Auto-consuelo Compasivo en la sesión}

Los siguientes apartados constituyen la especificación de la TAC de modo que pueda ser tanto aprendida y desarrollada como una competencia terapéutico, como evaluada en términos de la calidad de su ejecución (ya sea en contextos clínicos puros, formativos o de investigación). En ese sentido, los siguientes apartados pueden ser considerados como la estructura básica de un manual de entrenamiento, al que tan solo le faltarían ejemplificaciones y transcripciones de fragmentos de terapia que por razón de la naturaleza de este trabajo, no pueden ser desarrolladas aquí. por otra parte, el concepto de "manual" en el marco de la Terapia Focalizada en la Emoción (TFE) se refiere fundamentalmente a "manual de entrenamiento", y no a manual de aplicación. El trabajo en TFE se apoya en la sintonización empática con el cliente y en la facilitación de su proceso experiencial, basada en la formulación progresiva de sus estados internos (Goldman y Greenberg, 2014). Y en ese sentido, todo modelo de proceso y toda especificación de operaciones del terapeuta en relación con los estados internos del cliente -como las que se ofrecen aquí-, quedan condicionadas a su validación por parte de éste (el cliente como experto en su propia experiencia). Así mismo, han de servir como un mapa o guía (nunca equivalente al territorio), que facilite la mejor ejecución -según criterios empíricos de eficacia- a través de: (a) la mayor responsividad: afectación de la conducta del terapeuta por un contexto que no es dado ni previsible, sino que va emergiendo bidireccionalmente en la relación terapéutica momento a momento (Stiles, Honos-Webb y Surko, 1998) y (b) el máximo ajuste interactivo centrado en la persona a través de la sintonización empática, el diagnóstico de proceso y el equilibrio entre las actitudes de seguir y de guiar al paciente. Por todo ello, en este trabajo replicamos con la TAC las secciones que Greenberg et al. (1993) utilizaron para presentar sistemáticamente y manualizar las tareas terapéuticas originales de la TFE.

\section{A. La dificultad de procesamiento subyacente (¿Qué hay que cambiar?)}

El auto-consuelo se refiere a un proceso de regulación emocional en el que los clientes son tiernos, cariñosos y reconfortantes consigo mismos durante un estado emocional negativo intenso, de modo que esta experiencia transforma sus sentimientos. Conceptualmente, el trabajo de auto-consuelo es el antídoto de la autocrítica, aunque no funciona suprimiendo el proceso autocrítico, sino mediante la integración del dolor emocional que subyace en la voz crítica o en las experiencias no resueltas, ayudando entonces al cliente a acceder a recursos alternativos de auto-apoyo. Por tanto, el propósito del auto-consuelo es ayudar a los clientes a experienciar la validación y transformación de su sensación de sí mismos cuando ésta se organiza en términos de un esquema de emoción desadaptativo de estancamiento, dolor y desesperación, que hace colapsar el sí mismo (pasando por ejemplo de un sí mismo "carente de valor/imposible de amar" a un sí mismo "valioso/amable").

\section{B. El mancador para el auto-consuelo (oportunidades parala intervención)}

Si bien no existe un único marcador que nos informe de que el cliente se encuentra en disposición de trabajar con la TAC, la descripción fenomenológica transversal a todos los marcadores posibles se refiere a un estado de colapso o desmoronamiento del sí mismo, que sucede a un estado de angustia o desesperación desregulados. En general, en ese momento, el cliente puede experimentar:

a) una incapacidad para tolerar y regular la angustia, y entonces se ve inundado por ella, con un elevado grado de activación (e.g., en términos de vergüenza o miedo), o

b) una desesperación y un dolor intolerable (que le rompe o le hunde), ante una necesidad existencial no satisfecha (amor o validación), que se está reviviendo en ese momento. 
Tabla 3

Formas del marcador en la Tarea de Auto-consuelo Compasivo

\begin{tabular}{|c|c|c|}
\hline Marcador & Descripción & Ejemplo \\
\hline $\begin{array}{r}\text { Un sí mismo } \\
\text { desmoronado y débil }\end{array}$ & $\begin{array}{l}\text { Contacto con un fracaso interpersonal } \\
\text { o frente a una voz autocrítica que } \\
\text { producen un sentimiento de } \\
\text { desesperación, aislamiento, abandono, } \\
\text { vergüenza o falta de valía. }\end{array}$ & $\begin{array}{l}\text { "Hay algo que nunca he tenido [la } \\
\text { tristeza está en el nunca]... y es sentir } \\
\text { que yo soy bueno / o que estoy bien } \\
\text { para... [un otro significativo]". } \\
\text { "Es como si fuera incapaz de hacer } \\
\text { sentir segura a esa parte de mí, como } \\
\text { si esa parte estuviera siempre } \\
\text { preocupada [la tristeza está en el } \\
\text { siempre]." }\end{array}$ \\
\hline $\begin{array}{r}\text { Un sí mismo vulnerable } \\
\text { frente a un otro } \\
\text { implacable o maligno } \\
\text { en la silla vacía }\end{array}$ & $\begin{array}{l}\text { Fuerte dolor emocional y atascamiento } \\
\text { en la tarea (p.e., un repetido "No lo sé" } \\
\text { en el diálogo). }\end{array}$ & $\begin{array}{l}\text { "Ahora es como un dolor en el } \\
\text { estómago, duele pero nunca hay } \\
\text { nada." } \\
\text { "No sé qué puedo pedirle..." } \\
\text { [repetidamente]. }\end{array}$ \\
\hline $\begin{array}{r}\text { Fallos en las } \\
\text { estrategias habituales } \\
\text { para reactivar el sí } \\
\text { mismo colapsado en el } \\
\text { trabajo con las dos } \\
\text { sillas }\end{array}$ & $\begin{array}{l}\text { El fortalecimiento de la voz crítica o la } \\
\text { profundización en la silla del sí mismo } \\
\text { experienciante no surten efecto, y no } \\
\text { logran producir una reacción basada } \\
\text { en una necesidad y/o en el acceso a } \\
\text { una emoción primaria adaptativa. }\end{array}$ & $\begin{array}{l}\text { "Es... es imposible.. no puedo pedirle } \\
\text { nada. Según voy a abrir la boca ya veo } \\
\text { ese gesto, como mirándome sin } \\
\text { comprenderme y tan duro... y sé que } \\
\text { no hay nada que hacer..." }\end{array}$ \\
\hline
\end{tabular}

La angustia y la desesperación como emociones secundarias pueden ser validadas, a fin de que la persona acceda al sentimiento primario (e.g., el dolor del abandono) y que éste sea afirmado empáticamente (Elliott et al., 2004, pp. 130-140; Greenberg et al., 1993, pp. 311-321), con lo que habitualmente suele regularse y puede ser vivenciado. Al ocurrir esto en el contexto de la relación terapéutica, el cliente logra acceder a las necesidades subyacentes y articularlas de manera que nuevos pasos experienciales pueden ser dados (e.g., la expresión congruente de una necesidad como "necesito que me abraces y me digas que me quieres"). Luego, el acceso a una emoción primaria organiza el sí mismo de un modo más fuerte y adaptativo en ese momento (e.g., una madre responsiva en la otra silla en el trabajo con asuntos inacabados, puede decir: "Reconozco que no estuve ahí cuando me necesitabas, lo siento hija, pero te quiero aunque en su día, cuando tú lo necesitabas, no pude decírtelo"). Sin embargo hay ocasiones en que, bien por la fragilidad del sí mismo del cliente o a causa de otra condición, como es la confrontación con una figura maligna o implacable (real o interiorizada), éste se desmorona y entra en los estados de dolor desregulado que constituyen el marcador de la TAC (del cual, la Tabla 3 describe varias formas posibles).

\section{C. ¿Cómo ocurre el cambio? Modelo de resolución del proceso}

La Figura 1 es un esquema en el que se representa el proceso a través del cual evoluciona la TAC (diagrama de proceso), y que a continuación se describe en términos de las seis siguientes fases descritas inicialmente por Elliott (2010b):

1. Marcador: Sí mismo colapsado/atascado; activación emocional elevada.

Terapeuta: Refleja el marcador y propone la tarea. 


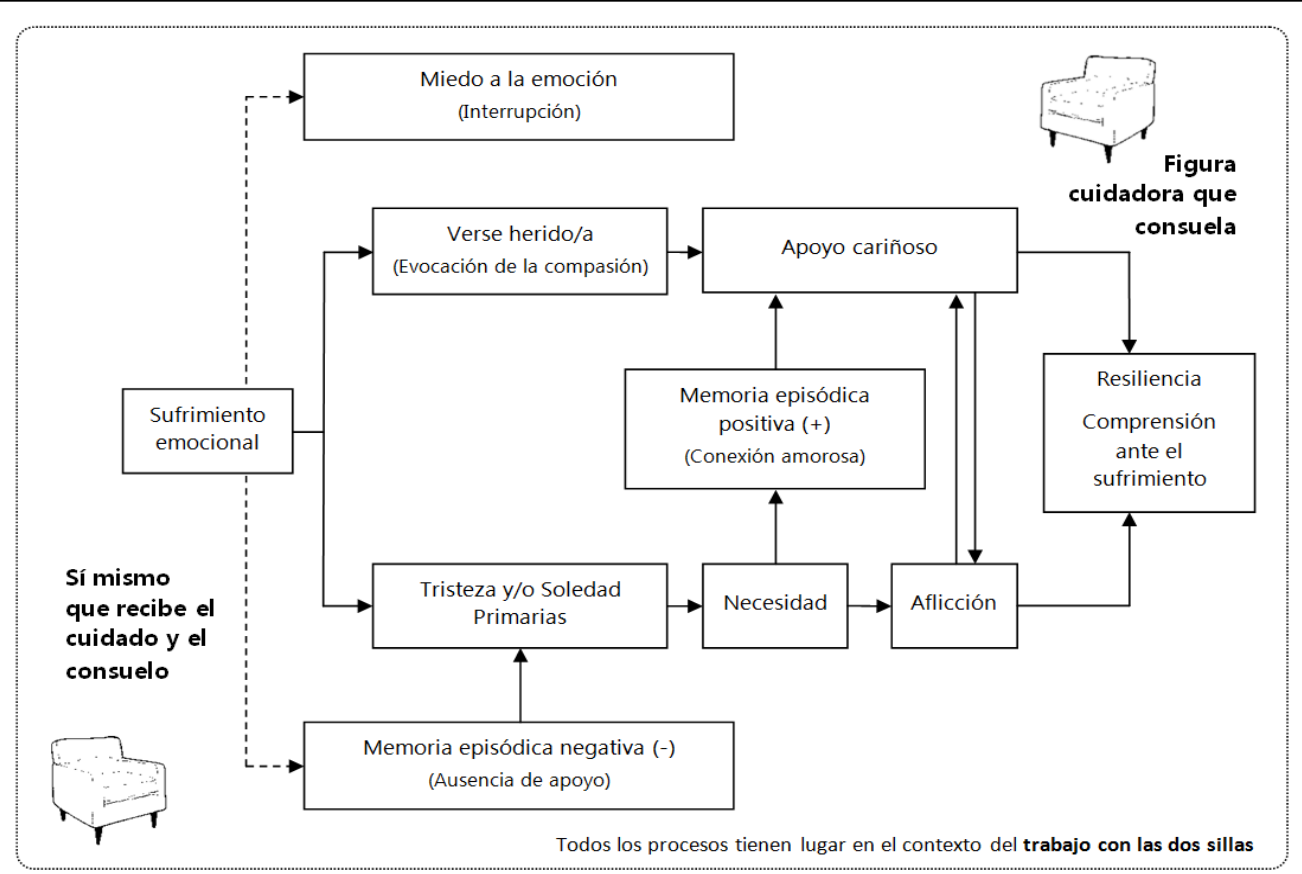

Figura 1. Diagrama de proceso de la Tarea de Auto-consuelo Compasivo; reproducido con permiso (Greenberg, 2011).

2. Iniciación de la tarea: Seleccionar y evocar el aspecto del sí mismo triste/asustado/desmoronado u otro.

Terapeuta: Propone una combinación sí mismootro para representar, adaptada a la necesidad. Entonces: (a) Pide al cliente que imagine el aspecto triste/asustado/colapsado, utilizando un lenguaje evocador; (b) cuando es posible, ayuda al cliente a identificar lo que necesita/quiere/desea, y (c) aporta empatía sólida hacia este aspecto y permite al cliente que experiencie realmente el estado de colapso/atasco antes de avanzar.

3. Ofrecer auto-consuelo: Representar el aspecto del sí mismo compasivo u otro similar.
Terapeuta: Anima al cliente en el rol de la persona compasiva para que ofrezca al aspecto colapsado lo que se desea para él o lo que necesita. Entonces modela el auto-consuelo y la actitud compasiva.

4. Resolución parcial: El cliente vive una alivio emocional/corporal.

Terapeuta: Dirige la escucha hacia el cambio en la sensación sentida corporalmente, que se produce en respuesta a la entrega de auto-consuelo compasivo y lo refleja. Entonces anima al cliente a estar con el nuevo sentimiento.

5. Resolución más completa: Cambio hacia una visión del sí mismo más positiva y empoderada.

Tabla 4

Escala de grados de resolución de la Tarea de Auto-consuelo Compasivo

1. El cliente se muestra como un sí mismo colapsado/atascado.

2. El cliente consigue superar el miedo a sentir o el miedo al rechazo, evoca la memoria episódica negativa, y llega a experienciar el dolor primario (tristeza o soledad).

3. El cliente que imagina el aspecto triste/asustado/colapsado y expresa lo que éste necesita desde esa posición.

4. El cliente representa el rol de la persona compasiva, se observa como alguien herido, siente compasión y ofrece al aspecto colapsado lo que desea para él o lo que éste necesita.

5. El cliente dirige la atención a la sensación sentida que se produce en respuesta al consuelo compasivo y vive una alivio emocional/corporal, que es la base de una visión del sí mismo más positiva y empoderada.

6. El cliente explora las posibilidades de extender ese sí mismo auto-compasivo o fortalecido a su vida. 
Terapeuta: Ayuda al cliente a explorar la nueva sensación del sí mismo más fuerte.

6. Llevar adelante: El cliente considera cómo extender este proceso en su vida.

Terapeuta: Ayuda al cliente a explorar posibilidades para llevara adelante ese sí mismo auto-compasivo o fortalecido en su vida.

En la Tabla 4 se recogen las seis descripciones que conforman la escala de grados de resolución de la TAC, y que se refieren a los logros del cliente a lo largo de este proceso.

\section{Operaciones y funciones del terapeuta en las distintas etapas del modelo}

El trabajo de facilitación de la TAC, frecuentemente requiere modular la alternancia entre marcos de participación (Sutherland et al., 2014), es decir entre el marco de la relación cliente-figura consoladora en la dramatización, y el marco de la comunicación clienteterapeuta. En ocasiones, los movimientos del cliente en búsqueda del contacto interpersonal con el terapeuta pueden ser vividos como "intentos auto-protectores" de salirse de la tarea, sin embargo, deben ser reencuadrados fundamentalmente como "esfuerzos de mantenerse en ella" buscando el reaseguramiento del terapeuta. Por eso éste debe manejarlos con delicadeza y tratando de identificar la dificultad emocional del instante y validarla empáticamente, sin enredarse en una argumentación racional sobre lo que ha de hacerse, ni tampoco aleccionando sutilmente al cliente en términos de cómo suele interrumpirse a sí mismo. Al contrario, una vez superado el bloqueo o acompañada la dificultad, normalmente basta una sencilla redirección animosa hacia el proceso, o una expresión de deseo genuino de volver a él juntos. Dos casos específicos de esta dificultad han sido identificados por Goldman y Fox (2010) en el análisis de tareas aplicado al autoconsuelo:

a) $\mathrm{Al}$ primero se le denominó "Protesta", y se refiere al momento en el que el cliente expresa no tener idea de cómo consolar al sí mismo.

b) El segundo fue denominado "Confrontación existencial", por la cual el cliente manifiesta una dificultad para ofrecerse el auto-consuelo, basada en la ausencia de experiencias de ese tipo en la vida y en las relaciones cercanas, lo cual despierta suspicacias o desconfianza. Por ejemplo: "Nunca he sido capaz de hacer algo así... nunca me lo hizo nadie a mí antes [...] Creo que ella [una niña universal], necesitaría recibirlo de alguien en quien alguna vez confió; de lo contrario, no se lo creería.

La Tabla 5 recoge una serie de sugerencias dirigidas al terapeuta para que desarrolle una actitud adecuada para facilitar el trabajo del cliente en la TAC, y para acompañar eficazmente las transiciones entre marcos de participación, al tiempo que el proceso discurre según el modelo de resolución.

\section{Validar el marcador}

Tras la aparición del marcador, en la primera fase el terapeuta refleja la dificultad y propone la tarea al cliente. El siguiente ejemplo clínico ilustra la aparición del marcador y su manejo por parte del terapeuta en el

Tabla 5

Sugerencias genéricas y actitudes de facilitación del proceso para el terapeuta

- Sé paciente; no trates de entrar demasiado rápido en el auto-consuelo. Asegúrate de que empleas el tiempo necesario para que el cliente evoque y experiencie la emoción dolorosa y el estancamiento/desmoronamiento.

- Asegúrate de que posees una conexión empática/compasiva realmente firme con este aspecto del sí mismo del cliente.

- Mantén una actitud de compasión genuina hacia el cliente.

- Recuerda que se trata de un proceso activo, guiando a través de una co-construcción colaborativa del ejercicio.

- Trata de mantener un estrecho seguimiento empático (afirmación empática de la vulnerabilidad y validación de la experiencia momento a momento).

- Sé paciente y mantén un ritmo adaptado a las necesidades de procesamiento del cliente.

- Realiza un manejo amable y empático de las transiciones entre marcos de participación para que el cliente se mantenga en la tarea. 
contexto del trabajo con la silla vacía para asuntos inacabados. En él una cliente (Ana, profesional de 33 años), trata de aclarar con su madre sus necesidades desatendidas en un momento vital clave (adolescencia), así como de comunicarle el dolor que eso le producía; sin embargo, la figura de la madre resulta inaccesible y la cliente entra en un estado de desconsuelo e impotencia que parece haberse cronificado:

C 2: No creo que pueda pedirle nada [a su madre]... me resulta imposible. Según lo pienso ya la estoy viendo reaccionar, ponerse rígida, y haciéndome sentir que no me entiende.. y esa es la sensación de siempre "No me entiendes, pero da igual"... [la expresión no verbal muestra hundimiento en el sillón y caída en la fuerza de la voz].

\section{Ayudar al cliente a conectar con la fuente de desconsuelo primordial}

Esto supone utilizar la exploración empática para evocar el aspecto del sí mismo que se desmorona y se angustia (reacciones afectivas secundarias) ante una situación o un otro significativo, y permitirle entonces acceder a sus emociones primarias (tristeza, soledad o dolor), de las que más tarde podrán emerger necesidades que serán expresadas en la situación de auto-consuelo. Siguiendo con el ejemplo anterior, el terapeuta refleja empáticamente la experiencia de impotencia ante una madre implacable, a la que la cliente no consigue llegar para hacerse entender:

T 7: Pero al lado de esa resignación y de esa dolorosa impotencia porque tu madre no llegue a ti, no se interese abierta y amorosamente por lo que a ti te pasa (y lo que te pasaba cuando eras una adolescencia y empezabas a salir, y necesitabas también poder mirarte en ella)... al lado de eso está toda la tristeza porque eso no ocurriera, toda la sensación de soledad (de tener que salir al mundo y dejar de ser niña tú sola), y todo el miedo a no saber hacerlo bien porque a ella nunca le gustaba nada de lo que hacías [en el relato anterior hay una historia de invalidación constante de sus gustos y aficiones].
El dolor primario (reacción directa a una situación), pudo ser adaptativo en los momentos de relación con la madre real a lo largo de la crianza. Sin embargo actualmente es desadaptativo en tanto es la reacción primaria ante situaciones en las que la persona ha de mostrarse como ella misma (natural y con sus necesidades de reconocimiento y validación ante otras personas). $\mathrm{Y}$ en esos casos viene organizándose de formas no auténticas para conseguir desesperadamente la validación de los demás. Cuando accede al diálogo con la madre que normalmente no fue validadora ni la amaba de modo incondicional en los momentos en los que así lo necesitaba, Ana se encuentra con un muro ante "un otro implacable", que sistemáticamente le hace entrar en un estado de desesperación y resignación dolorosa. En su relación con su madre real se ha acostumbrado a protegerse y a evitar esta experiencia limitando su exposición, aunque sigue quedando un sentimiento de resignación y de desconexión dolorosa cuando habla con ella, y más en el fondo, unos sentimientos de soledad y desamor no resueltos, que son los que deberán ser posteriormente beneficiarios de la experiencia de auto-consuelo.

En general podemos observar que lo que permite a las personas moverse de la emoción secundaria (miedo al dolor o resignación ante el dolor como en el ejemplo), es darse cuenta del proceso interruptor (evitación), y facilitar el acceso a la memoria episódica negativa, es decir, el acceso a los recuerdos de situaciones dolorosas de las cuales parte el estado emocional desregulado de colapso del sí mismo (en el ejemplo de Ana, el recuerdo de las situaciones de invalidación e imposibilidad de comunicación y conexión con la madre).

\section{Proponer la tarea}

El terapeuta crea un espacio cognitivo para la tarea, es decir, prepara mentalmente al cliente para hacer algo distinto en función del marcador, y para ello combina una argumentación racional sencilla con una explicación empática de lo que le propone hacer. 
T 8: Llegados aquí... quizá podríamos hacer algo para que la única opción no sea salir marcha atrás y encogida de hombros. Tú necesitas algo que tu madre no te puede o no te quiere dar... [comprueba reacción en la cliente].

C 9: [Abre los ojos con interés, aún en contacto con la desesperanza].

T 9: Se trataría de hacer algo distinto de lo que venimos haciendo; me gustaría ayudarte a que aquí en la sesión pudieras tener un diálogo con otra persona... con alguien que sí te pudiera dar el consuelo y el cariño que necesitas para romper esa resignación de "no me entiende... necesito que se baje hasta mí, me escuche y me comprenda sin juicios, cariñosa y abierta a escuchar y sentir lo que me pasa"...

C 10: Aja... [con interés].

T 10: Quizá podrías ser tú misma como mujer adulta... Como la persona sensata y sensible que eres ahora, capaz de escuchar y de tratar de comprender otras personas, y por supuesto a una niña o a una adolescente que necesita eso precisamente. ¿Crees que podríamos hacerlo si te voy ayudando?

C 11:Supongo... vamos. [y se endereza en la silla].

Estructurar la tarea y acceder a los estados internos del sí mismo que necesita consuelo

Tras ayudar al cliente a conectar con una sensación de desesperación y de angustia así como con la necesidad existencial asociada, y después de proponer la tarea, el terapeuta trata de estructurarla en el marco del trabajo con las dos sillas, y elige la combinación sí mismo-otro más adecuada para el caso. El terapeuta hace una propuesta y la reajusta de acuerdo con la retroalimentación del cliente. Elliott (2013) ha descrito cuatro combinaciones sí mismo-otro, que pueden ser utilizadas en el trabajo de auto-consuelo (Tabla 6). La naturaleza exacta de la combinación sí mismo-otro es negociada con el cliente a partir de las sugerencias hechas por el terapeuta. Una vez que la combinación sí mismo-otro es identificada, el trabajo de auto-consuelo se pone en marcha y el cliente va cambiando de silla entre el agente consolador y el sí mismo consolado.

Al proponer al cliente una combinación sí mismootro para representar adaptada a su necesidad, el terapeuta debe: (a) Pedir al cliente que imagine el aspecto triste/asustado/colapsado, utilizando un lenguaje evocador; (b) ayudarle a identificar lo que necesita/quiere/desea; (c) aportar empatía sólida hacia este aspecto y permitir al cliente que experiencie realmente el estado de colapso/atasco antes de avanzar, y (d) después pedirle que evoque la figura consoladora, para pasar a dialogar después con ella. Véase cómo sucede esto en el ejemplo clínico que venimos presentando:

T 11: Imagínate a ti misma con... ¿cuántos... 16 años?

C 12: Sí...

T 12: Si puedes, cierra los ojos... [respira] Imagínate a ti misma con 16 años,, ahí, en esa silla, queriendo enseñarle a tu madre una ropa que te has comprado... o queriendo contarle algo que te ha pasado en el instituto, o algo que quieres hacer y te apetece mucho...

Tabla 6

Combinaciones sí mismo-otro e instrucciones para estructurar la Tarea de Auto-consuelo

\begin{tabular}{r|l}
\hline Niño interno & Instrucciones \\
\hline Niño universal & Imagínate a ti mismo en la otra silla como un niño. \\
pequeño/herido/solitario/asustado y háblale. & Imagina a un niño asustado/solitario/herido en la otra silla y háblale. \\
(o un niño conocido) & Imagina a un amigo tuyo realmente cercano, que sea similar a ti, o que haya \\
& tenido experiencias parecidas a las tuyas y que estuviera sintiendo. \\
& exactamente lo mismo que tú. ¿Qué desearías/querrías para él/ella? ¿Qué le \\
& podría ayudar? ¿Qué podrías ofrecerle para ayudarle con todo eso? Inténtalo. \\
& ¿Le convence? Y si no, ¿qué podría hacerlo en su lugar? \\
& Imagina a tu padre/madre [o a otra persona importante para ti] en la otra silla, \\
& no como ellos son, si no como tu necesitaste o hubieras necesitado que \\
& fueran. Pideles lo que necesitas...
\end{tabular}




\section{13: [Se encoge reactivamente].}

T 13: ¿Puedes verla?

\section{[...]}

T 14: ¿Cómo se siente esa joven Ana? ¿Qué pasa dentro de ella?

C 14: Bueno... tiene un nudo en el estómago [pone su mano en su estómago instintivamente]...

$\mathrm{T}$ 15: Ya sabe lo que se le viene encima... y entonces...

C 15:Quiere irse corriendo...

T 16: Y si no se fuera corriendo sentiría otra vez que no hay manera...

C 16: Mmm [asiente aún con los ojos cerrados].

$\mathrm{T}$ 17: Pero tanto si se fuera corriendo como si se quedara, se llevaría consigo todo el dolor y la soledad de que su madre no la escuche.. no que le diga que sí a lo que ella quiere, sino que la escuche amorosamente.. que se acerque psicológicamente a ella...

Cabe añadir que en algunos casos es posible estructurar la tarea de modo imaginativo, en lugar de utilizar las sillas, especialmente en los casos en que esto resulte especialmente dificultoso para el cliente. Entonces se recurre al proceso que en el marco de la TFE ha sido denominado "reestructuración imaginativa" (Greenberg, 2002, p. 217), y que suplementa algunos de los potenciales activadores en el nivel emocional esquemático que poseen las dramatizaciones mediante un interrogatorio-guía que realiza el terapeuta, en el que incluye: (a) aspectos sensoriales (¿qué es lo que puedes ver, oír, oler o sentir en tu cuerpo? ), (b) emociones (¿qué es lo que sientes?), (c) pensamiento (¿qué es lo que hay ahora en tu cabeza?) y (d) conductuales (¿qué es lo que quieres hacer? o ¿qué es lo que está sucediendo?). Como sea, este paso requiere que el terapeuta ayude al cliente a activar su dolor y a permanecer en contacto con él, pues será esto lo que posteriormente le permita acceder al recurso interno del "yo consolador" (al verse herido y al evocar la memoria positiva de un cuidador), y pasar de la experiencia de la necesidad a la expresión del cuidado.
Facilitar la expresión de sentimientos y necesidades de la parte que necesita ser consolada

El terapeuta invita a la persona a ocupar la posición del sí mismo que necesita ser consolada y a expresar desde ahí y hacia la figura consoladora lo que siente y lo que necesita:

T 33: Hazle saber a Ana adulta que entonces te encoges... $\mathrm{Y}$ aunque puedas saber que tu madre te quiere, no lo puedes sentir... En ese momento sientes...

C 33: Que no me quiere... [completa la frase del terapeuta].

T 34: Dile "Entonces siento que mamá no me quiere..."

C 34: Entonces siento que mamá no me quiere... y me falta todo ese amor...

T 35: Que esté dentro de ti y que forme parte de tu seguridad y que te acompañe a todas partes...

C 35: ¡Y que no tenga que compararme con nadie por esa sensación de que no voy a estar a la altura para ella...!

T 36: "Así es como me siento... y eso es lo que necesito..." [evocando la acción de hablar a la Ana adulta].

C 36: Sí... eso es...

Ayudar a representar el aspecto compasivo del sí mismo y a ofrecer consuelo

Igual que es importante ofrecer empatía hacia el aspecto del sí mismo desconsolado y permitir que el cliente viva experiencialmente el estado de colapso/atasco antes de avanzar, se debe acompañar a la figura consoladora en el proceso de reconocer y validar estos sentimientos. En cierto modo, esto le legitima subjetivamente para dar el paso siguiente. En el ejemplo, el sí mismo compasivo comienza la expresión del consuelo con una profunda validación empática del sí mismo desconsolado:

C 37: [Cambia de silla].

T 38: Has visto y has oído a Ana... con 16, con 17, con ¿hasta cuántos años?

C 38: Hasta hace no mucho... Incluso hasta cuando me casé... Conozco la experiencia...

T 39: Díselo, pero antes mira a ver si puedes sentir su dolor y su impotencia... Mira a ver si la puedes hacer 
sentir comprendida y luego quizá darme u ofrecerle algo de lo que tanto necesita...

C 39: ¿Puedo cerrar los ojos al hablarle?

T 40: Sí, claro... adelante.

C 40: Sí... Es verdad... Mamá vive atrapada en todos sus miedos y a veces es muy egoísta y te quedas ahí sintiendo que está todo mal, que tienes que elegir entre hacer las cosas que a ti te gustan o hacer las cosas como ella dice... Porque si no está mal... Y aunque tú sepas lo que quieres, una parte de ti se queda dudando... no sólo de si estará bien o mal sino de si eso encajará con los demás y no puedes estar en lo que estás, sino que te andas volviendo a mirar si estás a la altura... y al final tienes la sensación desagradable de compararte con los demás y no te gusta.

Facilitar la expresión de consuelo y aprecio y/o a satisfacer la necesidad existencial (amor/validación)

En este momento se trata de ayudar a que el cliente, organizado según la voz de la figura de consuelo, ofrezca al aspecto colapsado lo que se desea para él o lo que éste necesita, así como sus sentimientos amorosos y compasivos. Todo ello en el marco temático del diálogo que se ha venido desarrollando. Como Pascual-
Leone y Greenberg (2005) especifican en su análisis sobre los estados afectivos y de significado propios de cada tarea terapéutica de la TFE, el estado de autoconsuelo -que se encuentra íntimamente ligado a una necesidad existencial-, posee una naturaleza reflexiva: el consuelo se ofrece como transformación de una compasión corporalmente sentida respecto al sí mismo evocado en la otra silla, en una expresión interpersonal. $\mathrm{Y}$ esto permite que el cliente se desplace hasta ocupar una posición de agencia, a través de la cual satisface la necesidad previamente expresada. La Tabla 7 recoge algunos ejemplos de lo que hacen los clientes para actuar y ofrecer el consuelo. En el ejemplo de Ana, el fragmento que presentamos a continuación sucede en el contexto de un auto-abrazo:

C 46: Anita... ¡me gustas como eres! Eres genial, eres creativa... todas esas aficiones locas que tienes son geniales... Me da igual si tardas un poco más que otras personas en aprender la cosas, porque ¡tú eres interesante!

T 47: Mmm...

C 47:Eres sensible... [lágrimas] y no me gusta que te sientas sola... Siento que no puedas hablar con mamá como necesitas... y siento que te sea tan triste... lo sé porque a mí también me pasa, pero...

Tabla 7

Ejemplos de acciones de consuelo de los clientes durante la tarea

Ejemplos de acciones de consuelo de los clientes durante la Tarea de Auto-consuelo Compasivo en TFE, extraídos del Manual de Clasificación de Estados Afectivos y de Significado de Pascual-Leone y Greenberg (2005).

Utilizando una manera reflexiva explícita

Consolando o cuidando del sí "mismo niño" desde el "sí mismo adulto" (Nadie veía lo que te pasaba, pero yo lo puedo ver, y sé que te sentías perdido y necesitabas que te dieran la mano; pues bien, aquí tienes mi mano...).

Charla con uno mismo en positivo (Sé que voy a estar bien...).

Imaginando el cuidado o el consuelo

Puedo imaginarme siendo abrazado... o yendo a un lugar seguro.

Puedo imaginar una situación mejor para mí en el futuro.

Sé que el amor de Dios está siempre ahí fuera...

Cuidado o consuelo atribuidos

Ofreciendo palabras de consuelo o de cuidado hacia uno mismo en la representación de la figura consoladora, por ejemplo hablando desde la otra silla como una madre (Voy a cuidar de ti...).

Imaginando la disculpa o el arrepentimiento de una figura que ofendió al cliente, de un modo que es equivalente a imaginar al otro adoptando una actitud cuidadora (Me siento fatal por haber estado ausente todo ese tiempo y por no haberte dicho nunca que te quiero, y me gustaría poder hacerlo...).

Reconociendo recursos existentes y recordando ejemplos autobiográficos actuales

Mi familia actual o mis amigos me quieren y cuidan de mí ahora.

[Alguien] me aprecia y hoy por hoy me da lo que necesité. Por ejemplo...

Mi hermana me quiere y mi marido tiene gestos amorosos conmigo que le salen del corazón; por ejemplo el otro día me vio triste y me trajo flores... 
T 48: Pero...

C 48:Pero [suspira] cuentas conmigo. Estoy a tu lado para decirte que te quiero. Quiero que sepas que te quiero.

\section{Facilitar la recepción vivencial del consuelo}

El terapeuta pide al cliente que ocupe la silla de la parte experienciante, y dirige su atención hacia la experiencia corporal sentida en el momento como reacción al mensaje de la parte consoladora. Ayuda así a que se forme una sensación sentida relativa a esa experiencia y anima al cliente a permanecer sin prisa con ese nuevo referente interno sentido corporalmente, así como con los sentimientos emergentes. En el caso del ejemplo no hay un cambio de silla, porque la transición ya se ha producido implícitamente en la experiencia del abrazo:

T 50: [Espera pacientemente, acompañando y protegiendo el silencio del abrazo].

C 50:[Lágrimas mientras se acaricia en el auto-abrazo].

T 51: Permítete sentir sin prisa el abrazo y lo que pasa dentro de ti... El modo en que lo recibe tu cuerpo junto con esas palabras: "Anita, te quiero".

C 51:[Concentrada se implica en la propuesta].

T 52:Todo ese amor incondicional...

C 52: Mmm [fuerte suspiro] Sí... Gracias... [Abre un ojo, y mira con complicidad y una sonrisa al terapeuta] Ay... [vuelve a suspirar].

T 53: ¿Se afloja algo ahí dentro?

C 53:Es como más espacio y como unas ganas grandes de estirarme.

T 54: ¡Pues adelante!

C 54: [Se estira en el sillón con un tierna sonrisa].

Ayudar al cliente a explorar la sensación del si mismo más fuerte

El terapeuta debe ahora ayudar al cliente a explorar cómo se siente, para que éste pueda simbolizar los cambios en su visión del sí mismo, esta vez más positiva y empoderada:

T 55: ¿Cómo te sientes Ana?
C 55: Bien... ligera, pero también... tierna.

T 56: Estás como estrenando una sensación.

C 56: Sí...

T 57:Habrá que protegerla...

C 57: Sí... pero es extraño, tampoco quiero esconderme. Es más acostumbrarme.

Ayudar al cliente a llevar adelante la nueva experiencia

En esta última fase, la intención del terapeuta es ayudar al cliente a llevar adelante o a extender el nuevo proceso o la nueva experiencia en su vida. Esta operación puede adoptar muchas formas, desde las más simples como "Puedes volver a este momento siempre que quieras" en el caso de ejemplo, a otras más estructuradas (e.g., desarrollando el mensaje de consuelo en una carta que se puede llevar en un bolsillo y que se puede volver a leer, o anclando el auto-abrazo como una experiencia de referencia, que puede ser repetida en un momento de dolor intenso).

$\mathrm{T}$ 58: Puedes volver a este momento siempre que quieras.

C 58: Lo que no quiero es irme de él.

T 59: Quedarte siempre sintiendo así.

C 59: Sí [muy serena].

$\mathrm{T}$ 60: Bueno, por hoy creo que nos merecemos un descanso...

C 60: Sí... Gracias por esto...

\section{Consideraciones finales para el entrenamiento}

Aunque la ilustración del apartado Operaciones y funciones del terapeuta en las distintas etapas del modelo es una adaptación didáctica de un proceso terapéutico real sucedido en la sesión de terapia, es necesario contar con nuevos registros (videograbaciones y transcripciones adecuadamente codificadas), que constituyan una base amplia de práctica a partir de la cual extraer evidencias, y poder obtener lo que Simons, Kuhsner, Jones y James (2003) -desde el campo de la 
Educación- llaman "generalización situada": el proceso por el cual los practicantes de una determinada disciplina, sólo aceptan evidencias a partir de las cuales modificar su práctica, si perciben una conexión clara con la situación en la cual la mejora tendrá lugar. Las generalizaciones situadas se tejen con ejemplos, circunstancias y experiencias particulares, a la vez que no separan los planteamientos teóricos generales de los ejemplos que les sirven de evidencia (Nemirovsky, 2002). En el caso del trabajo con manuales de entrenamiento clínico para tareas terapéuticas específicas en TFE, nos encontramos con una situación de este tipo, y por eso proponemos tanto un material como su enriquecimiento y mejora. A su vez, el recurso a los procesos de generalización situada ha sido propuesto también en el modelo emergente de Práctica Basada en la Evidencia en Psicoterapia (Barkham y Margison, 2007), como manera de romper la brecha entre clínicos e investigadores.

\section{Desarrollo de habilidades de percepción del terapeuta}

Goldman y Greenberg (2014) plantean que la formulación de caso en TFE en el nivel de procesos y en tiempo real se fundamenta en la noción de "diagnóstico de proceso", las cuales a su vez se apoyan inicialmente en las habilidades percepción del terapeuta, entendidas como su capacidad para identificar: (a) los micro-marcadores de proceso durante la exploración empática, (b) los marcadores de tarea, y (c) los micro-marcadores de estado durante el desarrollo de las tareas (que se apoyan en el conocimiento de los mapas de proceso y de las escalas de resolución de cada una). Por eso resulta de vital importancia entrenar a los terapeutas en el desarrollo de las habilidades de percepción que, en relación con la TAC, les permiten identificar y responder a estos marcadores sobre la marcha.

\section{Enfoque formativo y de entrenamiento}

El entrenamiento en la TAC se puede plantear como una ocasión para conocer y aprender a trabajar con una tarea experiencial en el marco de la TFE, o también como un recurso que puede ser integrado en repertorio de competencias de cualquier terapeuta, a fin de ayudar a los clientes a desarrollar mejores capacidades de autoregulación emocional. Sea cual sea el caso, el enfoque formativo en esta tarea requiere la práctica en role playing y la supervisión clínica, para lo cual se sugiere: (a) revisar la ejecución siguiendo el modelo proceso (Figura 1), (b) valorar el alcance de la intervención según la escala de resolución de la tarea (Tabla 4), y (c) utilizar la rúbrica de evaluación de la actitud del terapeuta durante la TAC, que hemos elaborado para este propósito (ver Apéndice). 


\section{Referencias}

Angus, L., Watson, J. C., Elliott, R., Schneider, K. y Timulak, L. (2015). Humanistic psychotherapy research 1990-2015: From methodological innovation to evidence-supported treatment outcomes and beyond. Psychotherapy Research, 25(3), 330347. doi: http://dx.doi.org/10.1080/10503307.2014.989290

American Psychological Association. (2006). Emotionfocused therapy over time [DVD]. Recuperado de http:/www.apa.videos/.

Barkham, M. y Margison, M. (2007). Practice-based evidence as a complement to evidence-based practice: From dichotomy to chiasmus. En C. Freeman y M. Power (Eds.), Handbook of evidencebased psychotherapies: A guide for research and practice (pp. 443-476). Chichester, UK: Willey.

Barnard, L. y Curry, J. (2011). Self-compassion: Conceptualizations, correlates, and interventions. Review of General Psychology, 15, 289-303. doi: http://dx.doi.org/10.1037/a0025754

Brazier, D. (1997). La condición necesaria es amor: Más allá del self en el enfoque centrado en la persona. En D. Brazier (Ed.), Más allá de Carl Rogers (pp. 6783). Bilbao, España: Desclée de Brouwer.

Caro, C. (2005). El concepto de "auto-organización" en la Psicoterapia Experiencial y de Proceso. Miscelanea Comillas, 63(123), 367-396.

De la Vega, I. y Sánchez Quintero, S. (2013). Terapia Dialéctico Conductual para el trastorno de personalidad límite. Acción Psicológica, 10(1), 4556. http://dx.doi.org/10.5944/ap.10.1.7032

Elliott, R. (2010a). Psychotherapy change process research: realizing the promise. Psychotherapy Research, 20(2), 123-135. doi: http://dx.doi.org/10.1080/10503300903470743
Elliott, R. (2010b, enero 14). Compassionate selfsoothing dialogue for stuck/collapsed self state [Mensaje en blog] Recuperado de http://peeft.blogspot.com.es/2010/01/compassionate-selfsoothing-dialog-for.html

Elliott, R. (2012). Emotion-Focused Therapy. En P. Sanders (Ed.), The tribes of the person-centred nation (pp. 103-130). Ross-on-Wye, UK: PCCS.

Elliott, R. (2013). Compassionate self-soothing for stuck/dysregulated anguish [Apuntes en PowerPoint no publicados]. Counselling Unit, University of Strathclyde.

Elliott, R. y Greenberg, L. S. (2007). The essence of Process-Experiential/Emotion-Focused Therapy. American Journal of Psychotherapy, 61(3), 241-254.

Elliott, R., Watson, J. C., Goldman, R. N. Y Greenberg, L. S. (2004). Learning Emotion-Focused Therapy: The process-experiential approach. Washington, DC: American Psychological Association.

Gendlin, E. T. (1997). El cliente del cliente: la vertiente del darse cuenta. En C. Alemany (Ed.), Psicoterapia Experiencial y Focusing: La aportación de E. T. Gendlin (pp. 279-290). Bilbao, España: Desclée de Brouwer.

Gilbert, P. (2009). The compassionate mind: A new approach to life's challenges. Londres, UK: Constable \& Robinson.

Gilbert, P. (2015). Terapia Centrada en la Compasión: Características distintivas. Bilbao, España: Desclée de Brouwer.

Goldman, R. N. y Fox, A. (2010, mayo). A task-analysis of selfsoothing in Emotion-Focused Therapy. Comunicación presentada en la $26^{\mathrm{a}}$ Reunión Anual de la Society for the Exploration of Psychotherapy Integration, Florencia, Italia.

Goldman, R. N. y Greenberg, L. S. (2010). Self-soothing and other-soothing in emotion-focused couples 
therapy. En A. S. Gurman (Ed.), Clinical casebook of couples therapy (pp. 255-280). New York, NY: Guilford Press.

Goldman, R. N. y Greenberg, L. S. (2013). Working with identityand self-soothing in emotion-focused therapy for couples. Family Process, 52, 62-82. doi: http://dx.doi.org/10.1111/famp.12021

Goldman, R. N. y Greenberg, L. S. (2014). Case Formulation in Emotion-Focused Therapy: Cocreating clinical maps for change. Washington, DC: American Psychological Association.

Greenberg, L. S. (1994). The investigation of change: Its measurement and explanation. En R. Russel (Ed.), Reassessing Psychotherapy Research (pp. 114-143). Nueva York: Guilford Press.

Greenberg, L. S. (2002). Emotion-Focused Therapy: Coaching clients to work through feelings. Washington, DC: American Psychological Association.

Greenberg, L. S. (2011). Skills training in a comprehensive set of tools for working directly with emotion. EFT Institute, Level 3. Apuntes multicopiados: Psychology Clinic, York University, Toronto.

Greenberg L. S. y Elliott, R. (2012). Corrective experience from a Humanistic-Experiential perspective. En L. G. Castonguay y C. E. Hill (Eds.), Transformation in psychotherapy: corrective experiences across Cognitive Behavioral, Humanistic, and Psychodynamic approaches (pp. 85101). Washington, DC: American Psychological Association.

Greenberg, L. S. y Pascual-Leone, J. (1995). A dialectical constructivist approach to experiential change. En R. Neimeyer y M. Mahoney, Constructivism in Psychotherapy (pp. 169-194). Washington, DC: American Psychological Assiciation.
Greenberg, L. S. y Watson, J. C. (2005). EmotionFocused Therapy for Depression. Washington, DC: American Psychological Association.

Greenberg, L. S., Rice, L. N. y Elliott, R. (1993). Facilitating emotional change: The moment-bymoment process. Nueva York, NY: Guilford Press.

Gross, J. J. (1999). Emotion and emotion regulation. En L. A. Pervin y O. P. John (Eds.), Handbook of personality: Theory and research ( $2^{\mathrm{a}}$ Ed., pp. 525552). Nueva York: Guilford.

Kabat-Zinn, J. (1993) Mindfulness meditation: Health benefits of an ancient Buddhist practice. En D. Goleman y J. Gurin (Eds.), Mind/Body Medicine (pp. 259-275). Yonkers, NY: Consumer Reports Books.

Kabat-Zinn, J. (2005). Coming to our senses: Healing ourselves and the world through mindfulness. Nueva York, NY: Hyperion.

Lane, R. D., Ryan, L., Nadel, L. y Greenberg L. S. (2015). Memory reconsolidation, emotional arousal, and the process of change in psychotherapy: New insights from brain science. Behavioral and Brain Sciences, $\quad 38, \quad 1-80$. doi: http://dx.doi.org/10.1017/S0140525X14000041

Linehan, M. M. (1993). Skills training manual for treating borderline personality disorder. Nueva York, NY: Guilford Press.

Neff, K. D. (2003a). Self-compassion: An alternative conceptualization of a healthy attitude toward oneself. Self and Identity, 2, 85-102. doi: http://dx.doi.org/10.1080/15298860390129863

Neff, K. D. (2003b). Te Development and validation of a scale to measure self-compassion. Self and Identity, 2, 223-250. doi: http://dx.doi.org/10.1080/15298860390209035

Nemirovsky, R. (2002). On guessing the essential thing. En K. Gravemeijer, R. Lehrer, B. van Oers y L. Verschaffel (Eds.), Symbolizing, modeling and tool 
use in Mathematics (pp. 233-256). Dordrecht, Holanda: Kluwer Academic Publishers.

Pascual-Leone, A. y Greenberg, L. (2005). Classification of AffectiveMeaning States. In A. Pascual-Leone, Emotional processing in the therapeutic hour: Why "the only way out is through" (Tesis Doctoral no publicada). York University, Toronto, Ontario, Canada.

Pascual-Leone, A., Greenberg, L. S. y Pascual-Leone, J. (2009). Developments in task analysis: New methods to study change. Psychotherapy Research, 19, 527542. doi: http://dx.doi.org/10.1080/10503300902897797

Pérez Álvarez, M. (2014). Las terapias de tercera generación como terapias contextuales. Madrid, España: Síntesis.

Shahar, B., Carlin, E. R., Engle, D. E., Hegde, J., Szepsenwol, O. y Arkowitz, H. (2012). A pilot investigation of emotion-focused two-chair dialogue intervention for self-criticism. Clinical Psychology \& Psychotherapy, 19, 496-507. doi: http://dx.doi.org/10.1002/cpp.762

Simons, H., Kuhsner, S., Jones, K. y James, D. (2003). From evidence-based practice to practice-based evidence: The idea of situated generalization. Research Papers in Education, 18(4), 347-364. doi: http://dx.doi.org/10.1080/0267152032000176855

Stiles, W. B., Honos-Webb, L. y Surko, M. (1998). Responsiveness in psychotherapy. Clinical Psychology-Science and Practice, 5(4), 439-458. doi: http://dx.doi.org/10.1111/j.1468-

2850.1998.tb00166.x

Sutherland, O., Peräkylä, A., y Elliott, R. (2014). Conversation analysis of the two-chair selfsoothing task in emotion-focused therapy. Psychotherapy Research, 24(6), 738-751. doi: http://dx.doi.org/10.1080/10503307.2014.885146
Thompson, R. (1994). Emotion regulation: A theme in search for definition. Monographs of the Society for Research in Child Development, 59 (2/3), 25-52. doi: http://dx.doi.org/10.1111/j.1540-5834.1994.tb01276.x

Whelton, W. y Greenberg, L. S. (2000). The self as a singular multiplicity: A process-experiential perspective. En J. Muran, Self-relations in the psychotherapy process (pp. 87-106). Washington, DC: American Psychological Association.

Yarnell, L. M. y Neff, K. D. (2013). Self-compassion, interpersonal conflict resolutions, and well-being. Self and Identity, 2(2), 146-159. doi: http://dx.doi.org/10.1080/15298868.2011.649545 


\section{Apéndice: rúbrica de evaluación de la actitud del terapeuta durante la TAC}

Tabla 8

Rúbrica de evaluación de la actitud del terapeuta durante la TAC (elaboración propia)

\begin{tabular}{|c|c|c|c|c|c|c|}
\hline 1 & $\begin{array}{l}\text { Muestra compasión genuina hacia el cliente } \\
\text { Las expresiones y reacciones del terapeuta respecto al dolor del cliente son } \\
\text { auténticas (nacen de su sentimiento real) y son expresadas genuinamente (suenan } \\
\text { creíbles). }\end{array}$ & & 2 & 3 & 4 & \\
\hline 2 & $\begin{array}{l}\text { Es activo/a en el proceso } \\
\text { Va guiando y favoreciendo una co-construcción colaborativa del ejercicio. No deja al } \\
\text { paciente solo, abandonado en el proceso. }\end{array}$ & & 2 & 3 & 4 & 5 \\
\hline 3 & $\begin{array}{l}\text { Realiza un estrecho seguimiento empático } \\
\text { Afirmación empática de la vulnerabilidad y validación de la experiencia momento a } \\
\text { momento. }\end{array}$ & & 2 & 3 & 4 & \\
\hline 4 & $\begin{array}{l}\text { Se mantiene presente y es paciente con el cliente } \\
\text { Se muestra paciente y mantiene un ritmo adaptado a las necesidades de } \\
\text { procesamiento del cliente. }\end{array}$ & & 2 & 3 & 4 & \\
\hline 5 & $\begin{array}{l}\text { Mantiene el equilibrio en la interacción } \\
\text { Maneja de modo amable y empático las transiciones entre marcos de participación } \\
\text { (cliente-cliente y cliente-terapeuta) a lo largo de la tarea, de modo que no hay saltos } \\
\text { abruptos ni reacciones de incomodidad en los momentos en que el cliente se retira } \\
\text { del diálogo de auto-consuelo y busca apoyo en el diálogo con el terapeuta. }\end{array}$ & & 2 & 3 & 4 & 5 \\
\hline 6 & $\begin{array}{l}\text { Valida y gestiona inteligentemente las emociones secundarias } \\
\text { Identifica y valida oportunamente las emociones secundarias (angustia y } \\
\text { desesperanza), de modo que permite al cliente dirigir la atención hacia las } \\
\text { emociones primarias. }\end{array}$ & & 2 & 3 & 4 & 5 \\
\hline 7 & $\begin{array}{l}\text { Es evocador en el momento de facilitar el acceso al dolor primario } \\
\text { Utiliza un lenguaje, una imaginería y un tono de voz evocadores, que ayudan al } \\
\text { cliente a sentir su dolor primario según múltiples dimensiones del esquema } \\
\text { emocional (sensorial, corporal, de significado, motivacional, etc.). }\end{array}$ & & 2 & 3 & 4 & 5 \\
\hline 8 & $\begin{array}{l}\text { Tolera el dolor del cliente y le ayuda a experienciarlo } \\
\text { Facilita el contacto del cliente con el dolor primario, gestionando la eventual evitación } \\
\text { experiencial y ayudándole a simbolizarlo (en términos de sentimientos y } \\
\text { necesidades). }\end{array}$ & & 2 & 3 & 4 & 5 \\
\hline 9 & $\begin{array}{l}\text { Mantiene el contacto y el diálogo entre partes } \\
\text { Ayuda al cliente a expresarse desde cada posición en el diálogo de auto-consuelo, } \\
\text { sin dar por hecho ningún turno y favoreciendo el contacto activo y consciente entre } \\
\text { partes. }\end{array}$ & & 2 & 3 & 4 & 5 \\
\hline 10 & $\begin{array}{l}\text { Acompaña y aprecia las nuevas sensaciones del sí mismo del cliente } \\
\text { Da espacio a la experiencia de ser consolado y ayuda al cliente a estar con ese } \\
\text { sentimiento, así como a experienciarlo y darle volumen en términos de las diversas } \\
\text { dimensiones del esquema de emoción (sensorial, corporal, de significado, } \\
\text { motivacional, etc.). }\end{array}$ & & 2 & 3 & 4 & \\
\hline
\end{tabular}


